$\xi=$ 离

\title{
A prospective study on causes and functional outcome of frozen shoulder
}

\author{
Sanjeev Mahajan ${ }^{1 *}$, Daksh Gadi $^{2}$, Rahul Gupta ${ }^{3}$, Saurav Singla ${ }^{3}$, Piyush Setia ${ }^{4}$, Som Gupta ${ }^{5}$, Raminder Kaur ${ }^{6}$ \\ ${ }^{1}$ Director and Head of Department, Orthopaedics and Joint Replacement, Fortis Hospital, Ludhiana \\ ${ }^{2}$ Fellow in Arthroplasty, Department of orthopaedics and joint replacement, Fortis Hospital, Ludhiana \\ ${ }^{3}$ Senior Resident, Department of orthopaedics and joint replacement, Fortis Hospital, Ludhiana \\ ${ }^{4}$ Resident, Department of orthopaedics, DMC, Ludhiana \\ ${ }^{5}$ Head of Department, Department of Physiotherapy, Fortis Hospital, Ludhiana \\ ${ }^{6}$ Physiotherapist, Department of Physiotherapy, Fortis Hospital, Ludhiana \\ *Corresponding author E-mail: dr_daksh@yahoo.com
}

\begin{abstract}
Backgroud: Frozen shoulder also called as adhesive capsulitis is a condition characterised by global limitation of humeroscapular motion resulting from contracture and loss of compliance of the glenohumeral joint capsule. Frozen shoulder is a common problem and results in frustrating debilitation for its sufferers. There can be many reasons for pain and stiffness of shoulder joint, so it is very important to differentiate between adhesive capsulitis and the other causes.

Objective: The main objective of the study was to evaluate the various modes of treatment for frozen shoulder and to identify various causes and associations of frozen shoulder in Indian population. To the best of our knowledge no prospective study has been done which have compared different treatment options in patients of adhesive capsulitis in Indian population.

Methods: The study was done on 75 patients which were divided into three groups, based on the mode of management i.e. conservatively with medication and physiotherapy, physiotherapy and intraarticular injection and arthroscopic capsular release. The range of movement and functional outcome was compared using Constant \& Murley score.

Conclusion: We found that patients undergoing conservative management showed benefits of various treatment options if the condition is diagnosed at an early stage but the results convincingly prove the advantages of arthroscopic capsular release in patients with chronic painful stiff shoulder or in failed conservative treatment.
\end{abstract}

Keywords: Adhesive Capsulitis; Arthroscopic Capsular Release; Frozen Shoulder

\section{Introduction}

Idiopathic adhesive capsulitis is a commonly recognised but poorly understood cause of painful and stiff shoulder. Frozen shoulder syndrome was first described by Duplay in 1872 (Duplay ES 1872). In 1934, Codman used the term Frozen shoulder to describe this condition (Codman EA 1934). Frozen shoulder is defined as slow onset pain near the insertion of deltoid muscle with loss of internal rotation initially followed by more than 50 percent loss of external rotation and abduction of less than 90 degrees (Bhargav D \& Murrell GA 2004).

Stiff shoulder is a common problem in our clinics and classically considered as self-limiting condition. Despite years of investigations, little agreement exists regarding its diagnosis, etiology, pathology and management.

The incidence of frozen shoulder is three percent in general population and is usually seen in a patient in 50-70yr age group. The pathoanatomy of stiff shoulder is not well established till now. MRI and arthroscopic evaluation has helped to evaluate the pathoanatomy in greater detail. Though many hypothesis were made to explain the etiopathology, none of those were found to be conclusive.

The pathology of frozen shoulder has been recently described as 'dupuytren's like' contracture of the coracohumeral ligament and capsule by Bunker and Anthony. This leads to loss of external rotation. A study done in Nuffield orthopaedic centre, Oxford, U.K. on 22patients concluded the pathology of frozen shoulder to a chronic inflammatory response with fibroblastic proliferation. Bruckner and Nye et al reported 25 percent incidence of frozen shoulder in neurosurgical patients who had suffered subarachnoid haemorrhage (Bruckner EE \& Nye CJ 1981).

Its exact pathophysiology is yet an unanswered question; postulated causes include autoimmune connective tissue disorder, recurrent haemarthosis, reactive arthropathy, crystal arthropathy, infection, trauma, algodystrophy, suprascapular nerve entrapment and rotator cuff degeneration.

Reidel was the first to suggest that the basic pathology of shoulder stiffness may be localised to the joint capsule (Reidel R 1916). Early investigators suggested adhesive capsulitis as either an inflammatory process or a fibromatosis. Neviaser identified perivascular infiltration, capsular thickening, contracture and fibrosis in biopsy specimens (Neviaser JS 1962). Simmonds noted some focal necrosis in the tight inelastic tissues around the joint and attributed it to tendon, contracture and tears of the rotator cuff (Simmonds FA 1949). Lundberg identified an increase in the density of capsular collagen and a pattern of glycosaminoglycan distribution and he linked to the repair reaction (Lundberg BJ 1969).

There are three stages that classically characterise the disease: freezing, frozen and thawing (Murnaghan JP 1990) the freezing or 
painful stage begins when a patient initially notices the onset of aching pain which often begins at night and persists during the day; and is unrelated to activity. This phase lasts somewhere in between two to nine months. The progressive stiffness or frozen phase lasts between 3 to 12 months. Stiffness progresses to limit shoulder motion in all planes. Activities of daily living are severely restricted. Pain is less than inflammatory phase which lasts for twelve to forty two months. This stage can be as short as 4 weeks especially with aggressive operative management (Harryman DT et al 2009)

Bilateral involvement ranges from $10 \%$ in general population to as high as $40 \%$ in insulin dependent diabetes mellitus (Binder AL et al 1984). A frozen shoulder rarely occurs in the same shoulder unless an injury or disease process predisposes the joint to repeated episodes of stiffness.

Risk factors include age group of 40-60yrs, with mean age of $55 \mathrm{yrs}$ in males and 52yrs in females (Lundberg BJ 1969) surgical intervention in or around the shoulder, distinct or minor trauma, immobility following injury, surgery or any painful source. Disuse is suspected to be a major cause and early rehabilitation is considered as best preventive measure. Diabetes mellitus has been found to be associated with frozen shoulder in $10-20 \%$ of patients in the study done by Bridgman. (Bridgeman JF 1972). Bilateral involvement can go upto $42 \%$ (Fisher $L$ et al 1986).

\section{Objective of study}

There are many modes of treatment given in literature for treating adhesive capsulitis, yet no consensus has been reached for the ideal treatment option. In our study we have compared various treatment options for adhesive capsulitis. Various studies have been done on the different types of treatment which include physiotherapy, manipulation under anaesthesia, intraarticular and periarticular injection, open as well as arthroscopic capsular release. To the best of our knowledge no prospective study has been done which have compared different treatment options in patients of adhesive capsulitis in Indian population.

\section{Material and methods}

All patients presenting with frozen shoulder were categorised according to the etiology of frozen shoulder and functional range was assessed in each of them.

Patients with global restriction in range of movements in shoulder with a documented restriction of glenohumeral and scapulothoracic motion of 100 degrees of abduction or less and less than $50 \%$ of external rotation with normal findings on true anteroposterior and axillary lateral radiographs of the glenohumeral were diagnosed as adhesive capsulitis.

All patients with history of fracture around the shoulder, glenohumeral arthritis, instability and recurrent dislocation of shoulder were excluded from the study.

Rotator cuff tendinopathy was eliminated based on physical findings including normal strength and lack of impingement signs. MRI was not routinely done as adhesive capsulitis is a clinical diagnosis.

All patients with confirmed adhesive capsulitis were given a trial of analgesics, oral steroids, drugs for neuropathic pain with physiotherapy or physiotherapy with intraarticular injections initially for six weeks which consisted of basic passive and active gentle, firm stretching exercises involving all the four quadrants of capsule. The glenohumeral joint was injected via posterior approach using traditional posterior arthroscopic portal landmarks utilising a 21 gauge spinal needle. The solution contained $3 \mathrm{cc}$ of $1 \%$ lignocaine and $80 \mathrm{mg}$ of depomedrol. Maximum off two inraarticular injctions were tried.

Arthroscopic capsular release was done in all patients with stiff shoulder refractory to non-operative treatment. Scope was inserted with standard portals followed by resection of rotator interval synovitis with a motorized shaver. Arthrocare wand was intro- duced and anterior capsule was first released through the anterior portal beginning just inferior to biceps tendon and continuing to the inferior edge of glenoid till 5 'o clock position. Superior middle and inferior glenohumeral ligaments were released after switching the portals. Axillary pouch contractures were resected using scissors taking care of the axillary nerve. Subscapularis muscle was released from its insertion. Scope was reinserted in sub acromian space and bursectomy was done. Full range of motion was achieved on table. Post operatively no sling was given to the patient and both active and passive exercises were adviced to the patient.

\section{Discussion}

A total of 75 cases were included in the study and the patients were grouped into three groups i.e. first group comprising of patients treated conservatively with medication and physiotherapy, second treated by intra-articular steroid injections and physiotherapy, third group comprised of patients treated operatively by arthroscopic capsular release. Each group comprised of 25 patients. The majority of patients were aged between 55 to $65 \mathrm{yrs}$ of age $(60 \%)$. The mean age was 58.3 years.

The number of males included in the study was 28 and the number of females was 47 with the male to female ratio of 1.67:1.

All patients were right hand dominant, left side was seen more commonly. Three patients had bilateral involvement. The average duration of symptoms prior to presentation was 8.8 weeks. Earliest presentation was two weeks after developing of symptoms and 4 months was the maximum time after which the patient presented to us (Table 1).

Table 1: Duration of Symptoms

\begin{tabular}{lll}
\hline \multicolumn{3}{c}{ Table 1: Duration of Symptoms } \\
\hline Duration of symptoms (in weeks) & Number of cases & Percentage \\
\hline 4 & 22 & 30 \\
6 & 12 & 15 \\
8 & 8 & 10 \\
10 & 3 & 5 \\
12 & 15 & 20 \\
16 & 15 & 20 \\
\hline
\end{tabular}

The association of frozen shoulder was also studied with diabetes as the most common associated comorbidity with $40 \%$ of the patients. Among others were heart disease, stroke, cervical degenerative disease and thyroid disorder. $30 \%$ of the patients were having idiopathic frozen shoulder (Table 2).

Table 2: Comorbidities Associated with Frozen Shoulder

\begin{tabular}{lll} 
Comorbidities & No. of cases $(\mathrm{n}=20)$ & percentage \\
\hline Diabetes & 30 & 40 \\
Heart disease & 7 & 10 \\
Stroke & 4 & 5 \\
Cervical degenerative disease & 7 & 10 \\
Thyroid disorder & 4 & 5 \\
Idiopathic & 23 & 30 \\
\hline
\end{tabular}

Improvement in range of motion

The improvement in range of motion was analysed at six months of follow up. The average range of motion pre intervention and post intervention were noted as follows (Table 3 ).

Table 3: Comparison of ROM Pre and Post Intervention

\begin{tabular}{lll}
\hline MEAN ROM +/- & PRE- & POST- \\
SD & INTERVENTION & INTERVENTION \\
\hline Abduction & $56.25+/-11.087$ & $145+/-9.129$ \\
Forward flexion & $60+/-8.165$ & $147.50+/-5.00$ \\
External rotation & $16.25+/-2.500$ & $53.75+/-4.787$ \\
Internal rotation & Buttocks & D 12 \\
\hline
\end{tabular}

The mean improvement was $88.75+/-6.29$ degrees (range 80.00 to 95.00 ) for abduction, $87.50+/-5.00$ degrees (range 80.00 to 90.00) for forward flexion and 10 spinous level for internal rotation and $37.50+/-6.45$ degrees (range 30.00 to 45.00 ) for external rotation. 
Improvement in range of motion in cases with medication and physiotherapy.

Table 3: Improvement in ROM with Medication and Physiotherapy

\begin{tabular}{lll}
\hline Mean ROM +/- SD & Pre-intervention & Post-intervention \\
\hline Abduction & $58.57+/-21.157$ & $95.71+/-31.547$ \\
Forward flexion & $66.43+/-18.420$ & $102.14+/-27.058$ \\
External rotation & $16.43+/-2.440$ & $31.43+/-5.563$ \\
Internal rotation & Buttock & L5 \\
\hline
\end{tabular}

The mean improvement was $47.22+/-22.10$ degrees (range 30.24 to 64.21 ) for abduction, $43.33+/-23.98$ (range 24.90 to 61.70 ) for forward flexion, and 5 spinous process level for internal rotation and $37.50+/-6.45$ (Table 3 )

Improvement in range of motion in cases with physiotherapy and intraarticular injections (Table 4)

Table 4: Improvement in ROM with Physiotherapy and Intraarticular Injections

\begin{tabular}{lll}
\hline Mean ROM +/- SD & Pre-intervention & Post intervention \\
\hline Abduction & $58.89+/-9.610$ & $106+/-22.608$ \\
Forward flexion & $67.22+/-11.211$ & $110.56+/-26.745$ \\
External rotation & $15.00+/-5.00$ & $30.00+/-5.00$ \\
Internal rotation & Buttocks & L5 \\
\hline
\end{tabular}

Improvement in range of motion in cases undergoing arthroscopic capsular release (table 5).

Table 5: Improvement in Range of Motion in Cases Undergoing Arthroscopic Capsular Release

\begin{tabular}{lll}
\hline Mean ROM +/- SD & Pre-intervention & Post intervention \\
\hline Abduction & $56.25+/-11.087$ & $145+/-9.129$ \\
Forward flexion & $60.00+/-8.165$ & $147.50+/-5.00$ \\
External rotation & $16.25+/-2.500$ & $53.75+/-4.787$ \\
Internal rotation & Buttocks & D12+ \\
\hline
\end{tabular}

Comparison of range of motion in the three groups.

Table 6: Comparison of Range of Motion in the Three Groups

\begin{tabular}{llll}
\hline & $\begin{array}{l}\text { Medications and } \\
\text { physiotherapy }\end{array}$ & $\begin{array}{l}\text { Intraarticular in- } \\
\text { jections and phys- } \\
\text { iotherapy }\end{array}$ & $\begin{array}{l}\text { Arthroscopic } \\
\text { capsular release }\end{array}$ \\
\hline $\begin{array}{l}\text { Gain in } \\
\text { abduction } \\
\begin{array}{l}\text { Gain in } \\
\text { forward } \\
\text { flexion }\end{array}\end{array}$ & 47 & 37 & 88 \\
$\begin{array}{l}\text { Gain in } \\
\text { external } \\
\text { rotation }\end{array}$ & 15 & 35 & 87 \\
$\begin{array}{l}\text { Gain in } \\
\text { internal } \\
\text { rotation }\end{array}$ & $1-2$ spinous & 15 & 37 \\
\hline
\end{tabular}

So on comparing the three modalities, there was a gain of $88 \mathrm{de}$ grees of abduction with arthroscopic release as compared to 47 degrees and 37 degrees with the other two modalities. There was a gain of 87 degrees of forward flexion with arthroscopic capsular release as compared to 43 degrees and 35 degrees with other two. The external rotation also improved to 37 degrees with arthroscopic capsular release as compared to 15 degrees each with other two methods. Same was seen with internal rotation which saw a gain of 10 spinous process as compared with the other two interventions (table 6).

Thus to conclude in our series patient undergoing arthroscopic release for stiff shoulder showed better range of motion on follow up.

Improvement was assessed by Constant and Murley score (CM Score) in the three modalities of treatment.

For patients undergoing arthroscopic capsular release the mean pre op CM score of $26.00+/-5.8$ increased to $67.25+/-1.7$ post operatively, thus showing an improvement of $41.25+/-4.79$.

Whereas in patients undergoing physiotherapy alone and patients undergoing physiotherapy with intra-articular injections showed improvement of just $19.14+/-3.93(26.71+/-6.34$ to $45.86+/-$ 9.51) and $23.56+/-5.66(30.33+/-4.61$ to $53.89+/-9.3)$ respectively.

\section{Conclusion}

Frozen shoulder even though considered an enigma and a difficult problem to manage with various modalities of treatment described in literature. In our study we found that patients undergoing conservative management showed benefits of various treatment options if the condition is diagnosed at an early stage but the results convincingly prove the advantages of arthroscopic capsular release in patients with chronic painful stiff shoulder or in failed conservative treatment options.

But an initial trial of conservative management should always be tried in all patients because the condition is self-limiting in majority of cases and a satisfactory functional outcome may be achieved with treatment like physiotherapy or physiotherapy and intraarticular injections. The predictability of results with arthroscopic release in properly selected patients is beyond doubt. Internal rotation is better achieved with arthroscopic capsular release which may not be achieved with conservative management alone. An orthopaedic surgeon should have high index of suspicion for the increased chances of axillary nerve injury during the surgery and increased rates of dislocation post operatively.

\section{References}

[1] Duplay ES. De la peri-arthrite scapula-humerale ET des raideurs de 1'epaule qui en sont la consequence. Arch gen med. 1872;2:513542

[2] Codman EA. The shoulder. Boston, MA Thomas todd Company. 1934:216-224

[3] Bhargav D, Murrell GA. Shoulder stiffness: Diagnosis. Aust fam physician 2004 Mar; 33(3): 143-7

[4] Bruckner EE, Nye CJ. A prospective study of adhesive capsulitis of the shoulder in a high risk population. QJ Med 1981; 50: 191204

[5] Reidel R. Die Versteifung des shultergelenkes durch hangenlassen des armes. Munschen Med Wschr. 1916; 63: 1397

[6] Neviaser JS. Arthrography of the shoulder joint: study of the findings in adhesive capsulitis of the shoulder. J Bone Joint Surg Am. 1962; 44-A:1321-1359

[7] Simmonds FA. Shoulder pain with particular reference to the frozen shoulder. J Bone Joint Surg Br. 1949; 31B:426-2

[8] Lundberg BJ. The frozen shoulder. Clinical and radiological observations. The effect of manipulation under general anaesthesia. Structure and glycosaminoglycan content of the joint capsule. Local bone metabolism. ActaOrthop Scand Suppl. 1969; 119:1-59 http://dx.doi.org/10.3109/ort.1969.40.suppl-119.01.

[9] Murnaghan JP. The frozen shoulder. In Rockwood CA, Matsen FA $3^{\text {rd }}$ editors. The shoulder. $1^{\text {st }}$ edition (vol2). Philadelphia. Saunders Elsevier; 1990: 837-862

[10] Harryman DT, Lajarus MD, Rozencwaig R. The stiff shoulder. In Rockwood CA, Matsen FA $3^{\text {rd }}$, editors. The shoulder. $4^{\text {th }}$ edition (vol 2). Philadelphia. Saunders Elsevier; 2009: 1064-1112

[11] Binder AL, Bulgen DY, Hazleman BL, Roberts S. Frozen shoulder: a long term prospective study. Ann Rheum dis 1984; 43:361364 http://dx.doi.org/10.1136/ard.43.3.361.

[12] Bridgeman JF. Periarthritis of the shoulder and diabetes mellitus. Ann Rheum Dis. 1972; 31:69-71 http://dx.doi.org/10.1136/ard.31.1.69.

[13] Fisher L, Kurtz A, Shipley M. Association between cheiroarthropathy and frozen shoulder in patients with insulin dependent diabetes mellitus. $\mathrm{Br} \quad \mathrm{J}$ Rheumatol. 1986; 25:141-146. http://dx.doi.org/10.1093/rheumatology/25.2.141. 